\title{
The Influence of Sound Awareness on the Level of Sound Generated during Nursing Activity in a Pseudo-Ward
}

\author{
Tomoko Shimoda', Maria Asai ${ }^{2}$, Rei Yoshida ${ }^{3}$, Ririko Aoki' ${ }^{4}$, Rika Yano ${ }^{1}$ \\ ${ }^{1}$ Faculty of Health Sciences, Hokkaido University, Sapporo, Japan \\ ${ }^{2}$ Former Department of Health Sciences, School of Medicine, Hokkaido University, Sapporo, Japan \\ ${ }^{3}$ Sapporo Minami-Sanjo Hospital, Sapporo, Japan \\ ${ }^{4}$ MEGMILK SNOW BRAND Co., Ltd., Tokyo, Japan \\ Email: tshimoda@hs.hokudai.ac.jp
}

Received 22 February 2016; accepted 16 April 2016; published 19 April 2016

Copyright (C) 2016 by authors and Scientific Research Publishing Inc.

This work is licensed under the Creative Commons Attribution International License (CC BY).

http://creativecommons.org/licenses/by/4.0/

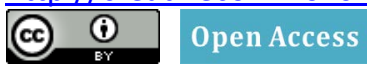

\begin{abstract}
Sound generated in a ward can be classified into 1) generated by medical staff, 2) generated by patients and their visitors, and 3) others, such as from in-hospital broadcasting microphones and nurse calls. Among these sounds, the incidence from medical staff, in particular, is reportedly high. The study objective is to investigate whether sound awareness is effective in regulating the sound environment even in a busy situation, such as in a real clinical setting, and to examine the extent to which sound awareness affects sound level. Nursing students were asked to perform a series of nursing activities in a pseudo-ward, and the changes in the sound level generated during the nursing activities with or without time and sound awareness were examined. Under varying experimental conditions, the sound and time levels associated with the nursing activities were measured in the following order: condition 1 , without sound or time awareness; condition 2 , with time awareness but without sound awareness; and condition 3 , with both sound and time awareness. The time to perform nursing activities was longer with sound awareness. However, when aware of time only, the sound level from nursing activities rose by $2.3 \mathrm{~dB}$, whereas when aware of both time and sound, the sound level dropped by $3.0 \mathrm{~dB}$. With both time and sound awareness, there is a distinct drop in the sound level from nursing activities, such as wagon handling, handling of items (trays, bowls), working at the sink, and opening and closing the microwave oven door. These results suggest that even in a pseudo-clinical setting it is possible to regulate the environmental sound through the environmental sound awareness of the medical staff, resulting in a drop in the sound level generated while performing nursing activities.
\end{abstract}

\section{Keywords}

Environmental Adjustment, Pseudo-Ward, Time, Sound Awareness, Nursing Activity 


\section{Introduction}

Within a hospital ward, various sounds are generated. According to the World Health Organization guidelines on sound, the normal level in a ward room should be lower than $30 \mathrm{~dB}$ and, in rooms where patients undergo treatment or examination, the level should be lower than $35 \mathrm{~dB}$ [1]. It is desirable for the patient's room to be a quiet environment; however, in medical settings, since various sounds are generated from the medical devices that are in use, regardless of the time of the day, it is difficult to comply with both of these guidelines [2]. In studies on Japanese hospitals, a sound level below $55 \mathrm{~dB}$ should be maintained within a ward [3]. Yet, there are instances of wards in overseas hospitals where sound levels above $50 \mathrm{~dB}$ have been reported [4] [5]. The upper limit of noise in rooms where patients undergo treatment or examination should be lower than the normal level. The medical staff within the study area will cause ambient noise levels to rise; to what degree is difficult to determine because rank correlation also suggests that the presence of patients and relatives, especially at visiting time, will cause ambient noise levels to rise as well.

Sound generated in a ward can be classified into 1) sound generated by medical staff, 2) sound generated by patients and their visitors, and 3) other sound, such as from in-hospital broadcasting microphones and nurse calls. Among these different sounds, there is a positive correlation between the noise level and the number of medical staff [4]. Moreover, based on studies regarding sound generated during nursing activities in a ward, the average sound level has been reported at $42.28 \mathrm{~dB}$, with levels rising most frequently above $70 \mathrm{~dB}$ during the day time (8:00 - 14:00) due to such activities [5]. Therefore, it is necessary for nurses to attempt to decrease the level of noise in a hospital environment; even in a busy and time-pressed clinical situation, if the sound level varies depending on the sound awareness of medical staff, we believe it likely that environmental sound levels can be regulated by encouraging sound awareness among medical staff.

The study objective was to elucidate the influence of sound awareness on sound level generated during nursing activities in a pseudo-ward. If it can be verified that the sound level is affected by sound awareness, we believe that the findings can be applied to medical staff sound awareness reforms and regulation of environmental sound levels.

\section{Materials and Methods}

\subsection{Participants}

The study period was August through November 2013. A total of 24 individuals who gave informed consent were selected from 180 sophomore-to-senior-level Japanese university students majoring in nursing and conversant in basic living assistance nursing technology.

\subsection{Design}

1) Experimental environment (Figure 1)

2) Activity setting

With reference to probable factors in ward noise that originate from medical staff as indicated in previous studies, the 6 categories of sound given below were measured:

(1) sound due to the handling of wagons,

(2) sound due to the handling of items (trays, bowls),

(3) sound due to activities at the sink,

(4) sound due to the use of microwave ovens,

(5) sound due to the handling of fences (curtains),

(6) sound due to the creating of nursing records.

A series of activities (activity A) involving these sounds in a ward environment was also determined (Table 1).

3) Experimental setting

The sound generated from each nursing activity was measured as the participants performed activity A under the following conditions in the given order:

Condition 1: without sound or time awareness (sound and time unaware),

Condition 2: with time awareness but without sound awareness (time aware),

Condition 3: with both sound and time awareness (sound and time aware). 


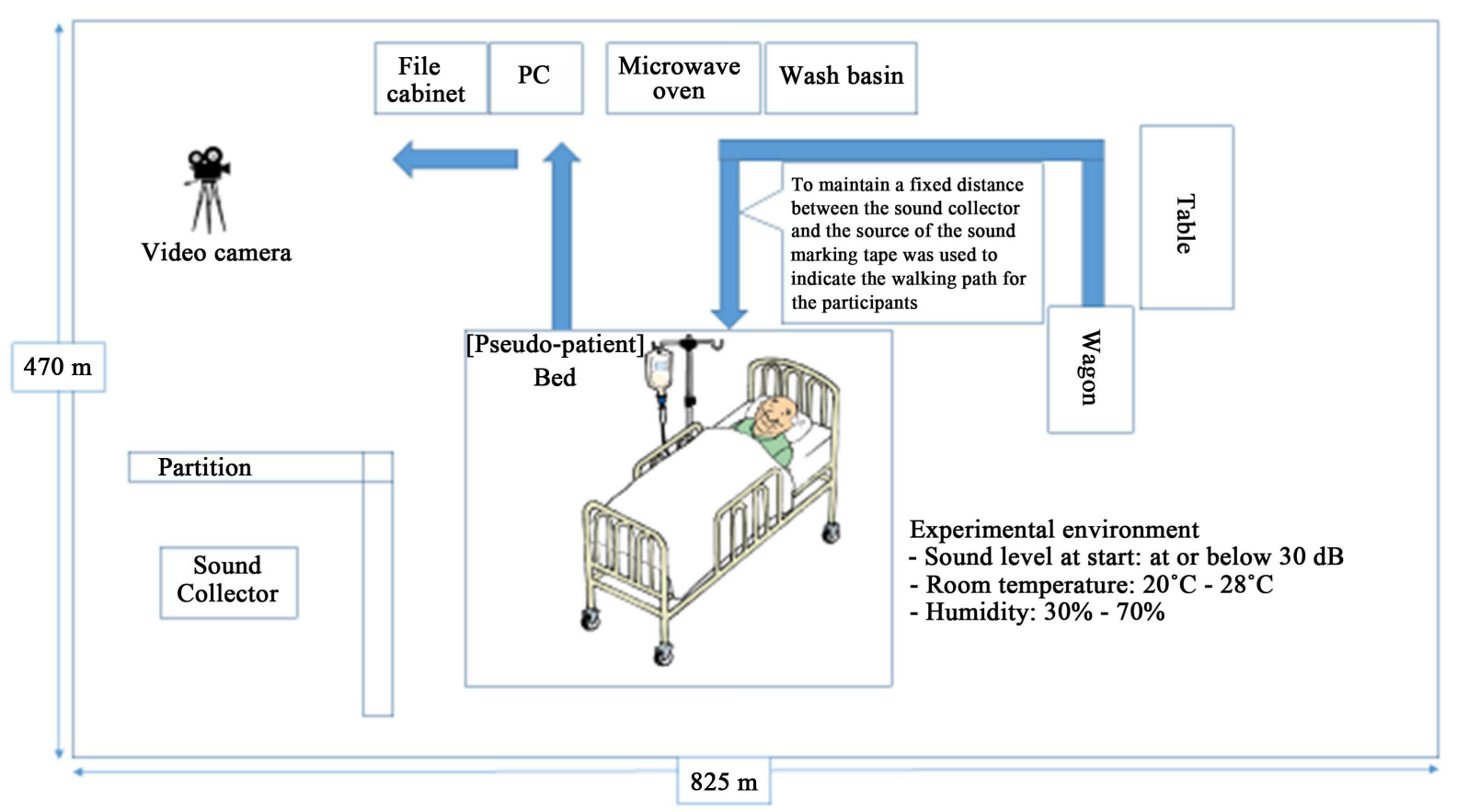

Figure 1. Experimental environment.

\subsection{Data Collection}

1) Sound generated due to nursing activity

The sound measurements were undertaken for nursing activities (Table 1) that generate sound. The sound level was measured using a sound level meter SL-1370 (measurement range 30 - $130 \mathrm{~dB}$, measurement accuracy (me dB, AS ONE Co., JAPAN), and measurements were taken at 0.5-second intervals using RS-232 analysis software (AS ONE Co., JAPAN).

2) Time needed in nursing activity

This was measured from video recordings using a digital video camera (HDC-SD9, Panasonic, JAPAN).

3) Awareness

The self-administered questionnaire focused on confirming participants' awareness of sound and time in relation to activity A; they were asked questions about the absence/presence of sound and time awareness that they experienced in the pseudo-ward.

\subsection{Experimental Procedure}

This experiment comprised two steps:

1) For each of the three experimental conditions [condition 1 (sound and time unaware), condition 2 (time aware), condition 3 (sound and time aware)], sound generated due to nursing activities was measured. The time required for each nursing activity was also measured.

2) To simulate a time-pressed clinical situation for experimental conditions 2 and 3, assuming nurse calls in approximately 10-second intervals, sound alerts were generated to stimulate sound awareness.

\subsection{Statistical Analyses}

To confirm that a specific measurement corresponds to a particular nursing activity, the video recordings were analyzed by several persons. For intermittent sounds, such as those due to the handling of items (trays, bowls), use of microwave ovens, handling bed guards, and keyboard typing, the maximum and minimum values were calculated, whereas for continuous sounds, such as those due to activities concerning wagons and sinks, the average was also calculated along with the minimum and maximum values. All data obtained were presented using average and standard deviation measures. In the statistical analysis, one-way analysis of variance was 
Table 1. Target activities for measurement of sound level from nursing activities.

Sound generated from nursing activities

Sound generated due to wagon handling

Sound generated due to handling of items (trays)

Sound generated due to handling of items (bowls)

Sound generated from using the sink

Sound generated from using the microwave oven

Sound generated from handling the bed rails

Sound generated due to activities

regarding recording nursing activities

\section{Target nursing activity for measurement}

Nursing activity (1) wagon handling

Nursing activity (2) put a tray in the wagon

Nursing activity (3) put a bowl in the wagon

Nursing activity (4) open the faucet and pour water into a bowl

Nursing activity (5) open and close the microwave oven

Nursing activity (6) set and detach bed rails

Nursing activity (7) type at the PC

Nursing activity (8) put away files

undertaken with time required and sound generated under the three experimental conditions. The average sound level generated during nursing activity under each of the experimental conditions was compared (using paired t-tests). The level of significance was established at $p<0.05$.

\subsection{Ethical Considerations}

Consent was obtained from the participants through written communication regarding the study purpose and method. We explained to the participants beforehand that participation was free and voluntary, consent could be withdrawn at any point, and the results would be anonymously recorded and would not be used for any purpose other than research. We also explained to the participants that any personal data available were viewable to the concerned person only and would be discarded after the study's completion. Since the experiment's purpose was to study the effect of sound awareness on sound generated, the term "sound environment" was not used in the explanations. This study was approved by the facility’s ethical review committee (approval no. 13 - 31).

\section{Results}

Among the 24 participants (all female, aged 20 - 22 years) who agreed to participate in the study, data from five participants were excluded because of equipment failures; therefore, data from 19 participants were analyzed.

\subsection{Experimental Environment}

Prior to commencing the experiments, the sound level in the experimental room was $39.6 \pm 1.9 \mathrm{~dB}$, the room temperature was $23.9^{\circ} \mathrm{C} \pm 2.0^{\circ} \mathrm{C}$, and the humidity was $45.2 \% \pm 11.6 \%$.

\subsection{Time Required}

Regarding the time required under each experimental condition, as shown in Figure 2, for condition 1 (sound and time unaware), it was $145 \pm 17 \mathrm{~s}$; for condition 2 (time aware), it was $113 \pm 18 \mathrm{~s}$; and, for condition 3 (sound and time aware), it was $123 \pm 22 \mathrm{~s}$. Thus, there was a significant reduction $(p<0.01)$ in the pseudo-ward conditions under condition 2 and 3 compared to condition 1 when participants were not aware of sound or time.

\subsection{Comparison of Sound Level under Each Experimental Condition}

As shown in Figure 3, regarding the average sound level for each experimental condition, when condition 1 $(52.2 \pm 1.8 \mathrm{~dB})$ was compared with condition $2(54.5 \pm 2.7 \mathrm{~dB})$, there was a significant increase of $2.3 \mathrm{~dB}$ for condition $2(p<0.01)$, whereas when condition $2(54.5 \pm 2.7 \mathrm{~dB})$ was compared with condition 3 (51.5 \pm 2.0$)$, a significant decrease of $3.0 \mathrm{~dB}(p<0.01)$ for condition 3 was observed.

\subsection{Comparison of Sound Generated from Nursing Activities under Each Experimental Condition (Table 2)}

For experimental conditions 1 and 2, the sound level for all activities under condition 2 was higher, but no 


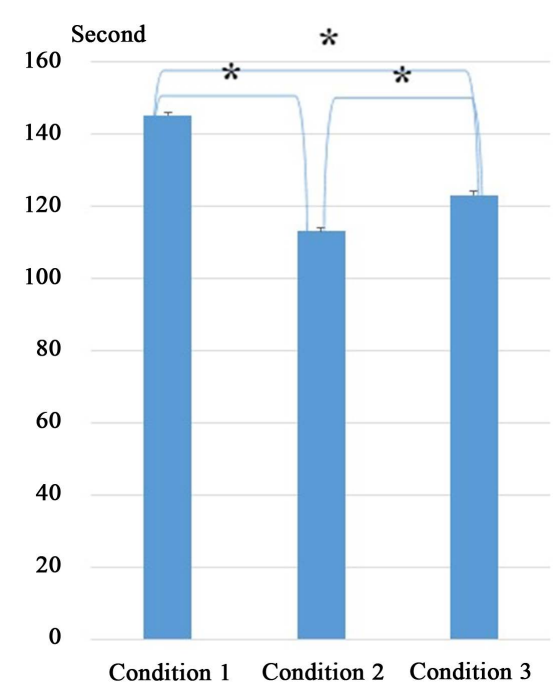

Figure 2. The time required under each experimental condition, Tukey Kramer ${ }^{*} p<0.01$.

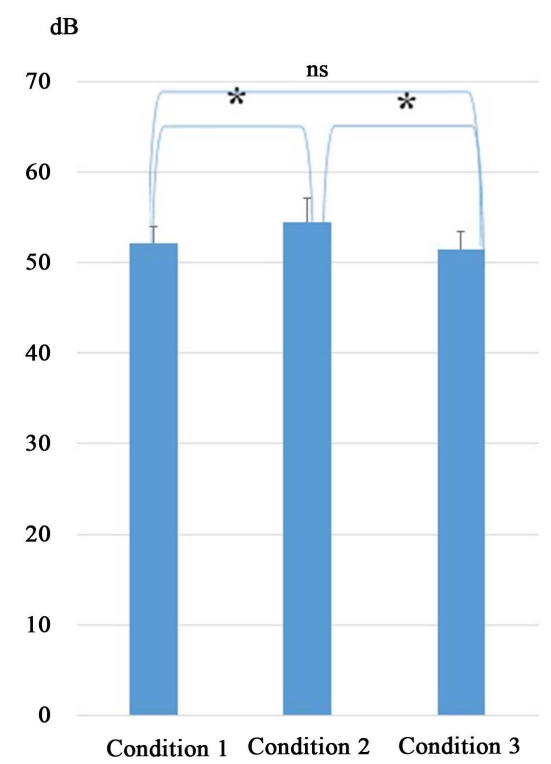

Figure 3. The average sound level for each experimental condition, Tukey Kramer ${ }^{*} p<0.01$.

Table 2. Variation of the average sound level from each nursing activity under each experimental condition $\mathrm{N}=19$.

\begin{tabular}{lccccc}
\hline \multirow{2}{*}{$\begin{array}{c}\text { Nursing } \\
\text { activity }\end{array}$} & \multicolumn{2}{c}{ Average sound level generated from each activity } & \multicolumn{2}{c}{ Significant difference } \\
\cline { 2 - 6 } & $\begin{array}{c}\text { Condition 1 } \\
\text { (Sound and time unaware) }\end{array}$ & $\begin{array}{c}\text { Condition 2 } \\
\text { (Time aware) }\end{array}$ & $\begin{array}{c}\text { Condition 3 } \\
\text { (Sound and time aware) }\end{array}$ & $\begin{array}{c}\text { Condition } \\
1 \text { vs. 2 }\end{array}$ & $\begin{array}{c}\text { Condition } \\
2 \text { vs. 3 }\end{array}$ \\
\hline (1) Wagon & 49.0 & 51.4 & 50.1 & n.s. & $p=0.0002$ \\
(2) Tray & 54.0 & 58.1 & 52.8 & n.s. & $p=0.0014$ \\
(3) Bowl & 58.3 & 60.5 & 56.0 & n.s. & $p=0.0002$ \\
(4) Sink & 51.4 & 54.1 & 51.7 & n.s. & $p=0.0003$ \\
(5) Microwave oven & 62.5 & 64.9 & 61.0 & n.s. & $p=0.0005$ \\
(6) Bed guards & 55.0 & 56.6 & 53.3 & n.s. & n.s. \\
(7) Typing & 46.1 & 47.1 & 46.1 & n.s. & n.s. \\
(8) Filing & 48.0 & 50.0 & 48.0 & n.s. & n.s. \\
\hline
\end{tabular}


statistically significant difference was observed. When the sound level was compared between condition 2 and condition 3, the changes in sound level for all nursing activities were lower for condition 3. In particular, for wagon handling, items (trays, bowls) handling, work at the sink, and using the microwave oven, the sound level was significantly lower $(p<0.01)$ for condition 3 compared to condition 2 .

\subsection{Presence/Absence of Time and Sound Awareness}

Responses were obtained from all participants targeted for analysis (response rate $100 \%$, valid response rate $100 \%)$.

Regarding measurements under condition 2, in response to the question "Were you aware of the time?" 10 participants (53\%) responded that they were very aware, while nine participants $(47 \%)$ responded that they were somewhat aware.

Under condition 3, regarding "Were you aware of the sound?" all participants responded that they were aware.

\section{Discussion}

\subsection{Experimental Environment Setting}

The environmental sound level was maintained below $41.4 \mathrm{~dB}$, closely similar to the general ward environment used in a previous study [3]. Since under condition 3 all participants were both time and sound aware, we believe that we were able to simulate a busy and time-pressed experimental environment. The temperature and humidity of the experimental room were maintained at the same level as in a general ward.

\subsection{The Effect of Time and Sound Awareness on the Sound Level Generated in Nursing Activities}

In studies with the ICU and emergency wards, reviewing the alarm settings and sound level in medical instruments can lead to a better sound environment resulting in improved sleep quality of patients [6] or reduction in noise level [7]. In a study describing staff consideration and alarm sound adjustments as likely key elements in improving the sound environment around patients, it has also been observed that speaking in a softer voice resulted in a difference of $16.78 \mathrm{~dB}$ compared to a louder voice for sounds generated by medical staff, and with proper efforts to lower the sound level, a reduction of $9.33 \mathrm{~dB}$ was achieved [8].

In this study, the experiment results suggest that although time awareness clearly leads to shortening of the activity time, sound awareness can also result in a reduction in sound level even in a time-pressed situation. Regarding the sound level generated in nursing activities, the activities that resulted in reduced sound level due to sound awareness were wagon activity, tray activity, bowl activity, sink activity, and microwave oven activity. Although the recommended sound level in a ward is below $30 \mathrm{~dB}$, the sound levels due to tray, bowl, and microwave oven use were high, exceeding $30 \mathrm{~dB}$ under condition 2 in the experiment. These three nursing activities were also the ones in which it was easier to reduce the sound level considerably by being sound aware.

For the sound involving trays between conditions 2 and 3, a difference of $5.4 \mathrm{~dB}$ was the largest among the activities observed, and we believe that this is an activity in which it is easier to reduce the sound level by being sound aware. Meanwhile, under condition 2, among the five activities in which the sound level could be lowered, wagon and sink activities recorded quite low sound levels. Although the sound level for these two cases does not exceed $30 \mathrm{~dB}$, we believe that these are activities wherein sound awareness can still lead to a reduction in the sound level. In particular, in the case of the sink, techniques such as holding the bowl closer to the faucet can suppress the sound of the water flow. According to previous studies, a noise level of approximately $10 \mathrm{~dB}$ can be attributed to sources, such as water flowing in lavatory sinks that patients consider unpleasant, and passing of wagons or catering cars during the daytime [9]. Therefore, we believe that based on the findings from our experimental data, ample room exists to devise sound reduction interventions.

Additionally, although the sound levels due to the handling of bed rails and nursing activity recording (typing and filing) were lower for condition 3 compared to condition 2, the difference was not statistically significant. These findings suggest that in these three activities, it is difficult to reduce the sound level even with awareness for sound environment adjustment.

The nursing activities selected in this study are routinely conducted in a ward, and are very likely to be per- 
formed concurrently by several medical staff. Because of this, even if there is no statistically significant difference due to a particular activity alone, when the activities overlap, the sound level is amplified, and events persist throughout the day, which can significantly affect the sound environment in a ward.

These results suggest that in cases of clinical settings where high sound levels are common due to activities performed in time-pressed situations, the sound awareness of the medical staff can lead to a reduction in the sound level generated from nursing activities.

\section{Conclusions}

1) Regarding the performance of a series of nursing activities in a pseudo-ward, being sound aware can reduce the sound level by approximately $3 \mathrm{~dB}$ compared to the sound unaware case.

2) In a pseudo-ward setting, being sound aware means that the sound level can be reduced in five stand-alone nursing activities, such as wagon handling, item (trays and bowls) handling, using the sink, and using the microwave oven.

\section{Limitations and Scope for Future Research}

As the experiments were conducted in a pseudo-ward, the simulated conditions may be different from real ward conditions where a mix of several sound sources exists. This paper mainly focused on the sound awareness of nurses. However, sound was not merely generated by nurses and the sound generated in a ward can be classified into 1) sound generated by medical staff, 2) sound generated by patients and their visitors, and 3) other sound, such as from in-hospital broadcasting microphones and nurse calls. This study was conducted in order to obtain basic data about the sound environment. Future studies must take into consideration the occurrence of other sounds in a real ward environment.

\section{References}

[1] Berglund, B., Lindvall, T. and Schwela, D.H. (1999) Guidelines for Community Noise Geneva: World Health Organization. http://whqlibdoc.who.int/hq/1999/a68672.pdf

[2] Darbyshire, J.L. and Young, J.D. (2013) An Investigation of Sound Levels on Intensive Care Units with Reference to the WHO Guidelines. Critical Care, 17, R187. http://dx.doi.org/10.1186/cc12870

[3] Yonenaga, T., et al. (1998) Comfort of Environmental Conditions in Dental Hospitals Evaluated by the Relation between a Questionnaire Survey of Patients and Environmental Measurements. Journal of Dental Health, 48, 325-334.

[4] Christensen M. (2005) Noise Levels in a General Surgical Ward: A Descriptive Study. Journal of Clinical Nursing, 14, 156-164. http://dx.doi.org/10.1111/j.1365-2702.2004.01040.x

[5] McLaren, E. and Maxwell-Armstrong, C. (2008) Noise Pollution on an Acute Surgical Ward. The Annals of the Royal College of Surgeons of England, 90, 136-139. http://dx.doi.org/10.1308/003588408X261582

[6] Hu, R., Hegadoren, K.M., Wang, X. and Jiang, X. (2015) An Investigation of Light and Sound Levels on Intensive Care Units in China. Australian Critical Care (in Press). http://dx.doi.org/10.1016/j.aucc.2015.08.001

[7] Way, R.B., Beer, S.A. and Wilson, S.J. (2014) What's That Noise? Bedside Monitoring in the Emergency Department. International Emergency Nursing, 22, 197-201. http://dx.doi.org/10.1016/j.ienj.2014.01.001

[8] Tegnestedt, C., et al. (2013) Levels and Sources of Sound in the Intensive Care Unit: An Observational Study of Three Room Types. Acta Anaesthesiologica Scandinavica, 57, 1041-1050. http://dx.doi.org/10.1111/aas.12138

[9] Kawasaki, M., et al. (2009) Noise Level and Night Annoyance in a Neurosurgical and Casualty Ward. Yamanashi Nursing Journal, 7, 5-11. 